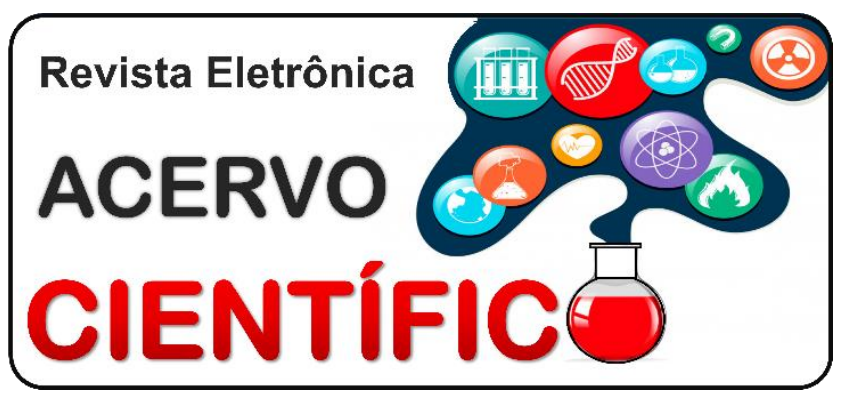

\section{REVISÃO BIBLIOGRÁFICA}

Recebido em: 2/2020

Aceito em: $3 / 2020$

Publicado em: 7/2020

\title{
Transfusões de sangue em unidades de terapia intensiva neonatal: uma revisão integrativa da literatura
}

Blood transfusions in neonatal intensive care units: an integrative literature review

Transfusiones de sangre en unidades de cuidados intensivos neonatales: una revisión integral de la literatura

Pâmela Cristina Furtado Costa ${ }^{1}$, Rubenilson Caldas Valois ${ }^{1 *}$, Marcia Helena Machado Nascimento1, Bárbara Alves Ruela de Azevedo Ruivo², Paula Christine Amarantes Justino Oliveira $^{2}$, Carla Nazaré Brito Duraens ${ }^{2}$, Glenda Roberta Oliveira Naiff Ferreira ${ }^{3}$, Michelly Tatiana Lima da Silva Batista ${ }^{1}$, Marcileide Silva da Costa ${ }^{1}$.

Resumo: Analisar as produções científicas nacionais e internacionais sobre transfusões de sangue que ocorrem no ambiente das unidades de terapia intensiva neonatal. Tratou-se de um estudo qualitativo descritivo, tipo revisão integrativa de literatura. As buscas foram realizadas nas bases de dados BDENF, LILACS e MEDLINE. Foram utilizados descritores em ciências da saúde e seus respectivos termos em inglês no Medical Subject Headings. Consideraram-se materiais com idiomas em Português, Espanhol e Inglês; contidos em periódicos científicos publicados nos anos de 2014 a 2019, sem restrições quanto ao nível de evidência. Para análise foram selecionados 08 artigos. Os dados foram processados no software IRAMUTEQ® e analisados descritivamente a partir da análise de similitude. Observou a formação de uma árvore com seis núcleos interligados, destacando nestes agrupamentos as seguintes palavras: "não", "prático", "necessário", "cuidados", "neonato", "recém-nascido", "transfusão", "relação", "estudo", "erro", "informação", "sistema", "pesquisa", "especialmente", "notificação", "discussão", "incidente", "adverso" e "dano". A temática transfusão de hemocomponentes em unidades de terapia intensiva neonatal é reconhecida como importante para terapêutica nos cuidados neonatais, no entanto, observa-se que há riscos inerentes a esta terapêutica. Sendo evidenciada nos textos a necessidade do monitoramento e controle do processo de reações adversas que ocorrem relacionados ao sangue.

Palavras-Chave: Recém-nascido, Transfusão de sangue, Publicações.

Abstract: To analyze the national and international scientific productions on blood transfusions that occur in the environment of neonatal intensive care units. This was a descriptive qualitative study, integrative literature review type. The searches were performed in the databases BDENF, LILACS and MEDLINE.

\footnotetext{
1 Universidade do Estado do Pará (UEPA), Belém - PA. *E-mail: rubenilsonvalois@gmail.com

2 Centro de Hemoterapia e Hematologia do Pará (HEMOPA), Belém - PA.

3 Universidade Federal do Pará (UFPA), Belém - PA.
} 
Descriptors in health sciences and their respective English terms were used in the Medical Subject Headings. We considered materials with languages in Portuguese, Spanish and English; contained in scientific journals published from 2014 to 2019, without restriction as to the level of evidence. For analysis, 08 articles were selected. The data were processed in the IRAMUTEQ ${ }^{\circledR}$ software and analyzed descriptively from the similarity analysis. It observed the formation of a tree with six interconnected nucleus, highlighting in these groupings the following words: "no", "practical", "necessary", "care", "newborn", "transfusion", "relationship", "study", "error", "information", "system", "research", "especially", "notification", "discussion", "incident", "adverse" and "damage". The theme of blood component transfusion in neonatal intensive care units have been recognized as important for therapy in neonatal care; however, there are risks inherent to this therapy. Being evidenced in the texts the need for monitoring and control of the process of adverse reactions that occur related to blood.

Keywords: Newborn, Blood transfusion, Publications.

Resumen: Analizar las producciones científicas nacionales e internacionales sobre transfusiones de sangre que ocurren en el entorno de las unidades de cuidados intensivos neonatales. Fue un estudio cualitativo descriptivo, tipo de revisión de literatura integrativa. Las búsquedas se realizaron en las bases de datos BDENF, LILACS y MEDLINE. Los descriptores en ciencias de la salud y sus respectivos términos en inglés se utilizaron en los encabezados de materias médicas. Consideramos materiales con idiomas en portugués, español e inglés; contenido en revistas científicas publicadas de 2014 a 2019, sin restricción en cuanto al nivel de evidencia. Para el análisis, se seleccionaron 08 artículos. Los datos se procesaron en el software IRAMUTEQ® y se analizaron descriptivamente a partir del análisis de similitud. Se observó la formación de un árbol con seis núcleos interconectados, destacando las siguientes palabras: "no", "práctico", "necesario", "cuidado", "recién nacido", "recién nacido", "transfusión", "relación", "estudio", "error", "información", "sistema", "investigación", "especialmente", "notificación", "discusión", "incidente", "adverso" y "daño". El tema de la transfusión de componentes sanguíneos en las unidades de cuidados intensivos neonatales se reconoce como importante para la terapia en la atención neonatal, sin embargo, existen riesgos inherentes a esta terapia. Se evidencia en los textos la necesidad de monitorear y controlar el proceso de reacciones adversas que ocurren relacionadas con la sangre.

Palabras clave: Recién nacido, Transfusión de sangre, Publicaciones.

\section{INTRODUÇÃO}

A terapêutica transfusional consiste em procedimento essencial para a equipe de saúde, sobremaneira, para os profissionais da enfermagem, de forma que cabe a estes o acompanhamento do processo transfusional (DUARTE ED, et al., 2010). Os procedimentos hemoterápicos usualmente referem-se a transfusões de frações específicas do sangue total, sendo estas frações as quais o paciente realmente necessita, evitando o uso de elementos sanguíneos desnecessários e que porventura possam causar reações adversas ao cliente. Procedimento que visa, principalmente, garantir o máximo de segurança ao paciente, assim como manter níveis satisfatórios de hemocomponentes nos bancos de sangue (SILVA KFN, et al., 2009).

Hemoderivados e hemocomponentes são provenientes da doação de sangue por um doador, que em território brasileiro encontra-se regulamentado pela portaria de consolidação n.5, do Ministério da Saúde do Brasil, onde aborda a proteção do doador, coleta, captação, estocagem, processamento, distribuição e transfusão do sangue, seus componentes e derivados e por regulamentos técnicos editados pelo Ministério da Saúde (MS) (MENDES NM e SOUZA SROS, 2011). Esses são por sua vez, produtos distintos, em que os que são gerados nos serviços de hemoterapia, um a um, partindo do sangue total por meio de processamento físico (centrifugação, congelamento) são os hemocomponentes. Já os produtos que são obtidos em escala industrial, por meio do fracionamento do plasma a partir de processos físico químicos são hemoderivados. 
O procedimento hemoterápico é um procedimento de responsabilidade multiprofissional, dividido em etapas que se interligam, fator este, que predispõe os profissionais dos serviços de saúde a cometerem frequentes erros associados a terapêutica. Tais falhas humanas, estão associadas diretamente ao procedimento seguro, assim como a segurança do cliente, isto definido pela Organização Mundial de Saúde, evento este que deverá ser minimizado ao máximo (BRASIL, 2014).

Para atuar nos procedimentos realizados no campo da hemoterapia, há a necessidade de profissionais de saúde treinados e capacitados, principalmente, quando na ocorrência de eventos adversos relacionados a tais terapias, fato este que poderá ocorrer durante ou até mesmo após o procedimento. Tornando a investigação um procedimento necessário e rigoroso conduzido por profissionais qualificados para prática com segurança (CHEREM EO, et al., 2016).

Para prática transfusional vários profissionais estão envolvidos, dentre estes, o enfermeiro atua embasado na Resolução n. 306, de 25 de abril de 2006, do Conselho Federal de Enfermagem (COFEN), a qual estabelece e delineia suas atribuições e responsabilidades quanto ao planejamento, coordenação, supervisão e execução, assim como a avaliação da prática hemoterápica em território nacional, visando desta forma assegurar a qualidade dos procedimentos em hemoterapia dispensados aos pacientes (BRASIL, 2014).

É importante a terapêutica transfusional (TT) no tratamento de doenças diversas, estruturando-se no ambiente prático, através de normas técnicas padronizadas, onde a segurança e qualidade do sangue e hemocomponentes devem ser assegurados. Esta prática transfusional é de ordem complexa que depende de uma equipe multiprofissional para garantir sua eficácia. Dessa forma, para que isto aconteça, tais profissionais precisam deter conhecimentos e habilidades além das suas competências, tendo assim conhecimento sobre a atuação de toda a equipe e do sistema em sua eficiência (SOUZA GF, et al., 2014).

O grupo de pacientes que mais usufrui dos hemocomponentes são os recém-nascidos que são hospitalizados nas unidades de terapia intensiva neonatal (UTIN) e a hemotransfusão neste grupo requer uma abordagem diferente da aplicada em adultos, por conta das seguintes características: imaturidade metabólica e imunológica; sensibilidade ao frio superior a do adulto; fisiologia hematológica peculiar; altos riscos de anóxia tecidual e patologias advindas do neonato (DINIZ EMA, et al., 2001).

Dentro da prática hemoterápica nas unidades de terapia intensiva neonatal utilizam-se critérios restritos na prática transfusional, visando menor exposição dos prematuros de extremo baixo peso aos múltiplos doadores (SHANNON KM, et al., 1995; MURRAY NA, 2004; KURTNER JM, 2003).

A terapia transfusional de hemocomponentes e hemoderivados integra um método relevante para a medicina atual. Quando aplicada corretamente em significativas condições de morbidade e/ou mortalidade, não havendo outras formas de prevenção ou controle, pode salvar vidas e garantir melhorias à saúde do recém-nascido (CHEREM EO, 2015). Como exemplo de terapia com hemocomponentes têm-se a exosanguíneotransfusão (EXT) que constituiu a primeira terapia bem-sucedida utilizada para o tratamento da icterícia neonatal grave. Embora a EXT seja considerada um procedimento seguro, não está livre de riscos e denota dados de mortalidade com variação entre 0,5 e 3,3\% (BOWMAN J e PHILIP AG, 2003).

Dentre os profissionais envolvidos na prática hemoterápicas em neonatos, temos os enfermeiros, onde é dever deste ter um olhar clínico, ter conhecimento dos cuidados a serem prestados durante todo o procedimento de transfusão, além da capacidade de atender o neonato quanto às eventuais reações transfusionais (TOREZAN G e SOUZA EM, 2015). Neste sentido, um estudo feito com enfermeiros em um hospital universitário do interior do estado de São Paulo, grande parte dos profissionais manifestou está pouco ou mal informada acerca do assunto, sendo que, a maioria soube informar somente benefícios da transfusão, porém não responderam quanto aos riscos que esta pode apresentar (FERREIRA O, et al., 2007).

Sem dúvida, o tratamento com hemocomponentes e hemoderivados é um processo que, apesar de sua correta administração e indicação precisa, respeitando os demais cuidados e normas preconizadas, permanece o risco associado a terapêutica. Por isso, a importância do cumprimento eficiente do ciclo 
hemoterápico, em que o processo se inicia com a seleção dos doadores, seguido de triagens sorológica e imunohematológica, processamento e fracionamento das unidades que foram coletadas, distribuição e ato transfusional, assim como a avaliação pós-transfusional (FREITAS BAC e FRANESCHINI SCC, 2012).

Dessa forma, este trabalho tem como objetivo analisar o perfil de produções científicas nacionais e internacionais sobre transfusões de sangue que ocorrem no ambiente das unidades de terapia intensiva neonatal.

\section{MÉTODOS}

O estudo teve uma abordagem qualitativa descritiva, do tipo revisão integrativa de literatura, permitindo a compreensão sobre a temática específica a partir de estudos independentes. A realização do estudo ocorreu por meio de seis etapas: formulação da questão de pesquisa; posteriormente definiram-se os critérios de inclusão e exclusão; escolha das informações que deveriam ser extraídas; análise das informações registradas com auxílio do programa de análise textual Iramuteq; interpretação e discussão dos resultados e apresentação da síntese das informações. Construiu-se o estudo a partir da seguinte questão norteadora: "Quais as principais produções científicas sobre as transfusões de sangue que ocorrem no ambiente das unidades de terapia intensiva neonatal?".

Para construção deste estudo consultaram-se as bases eletrônicas de dados Base de dados de Enfermagem (Bdenf), Medical Literature Analysis and Retrieval System Online (MEDLINE via PubMed) e Literatura Latino-Americana e do Caribe em Ciências da Saúde (LILACS via BVS). Para a busca utilizaramse os descritores em Ciências da Saúde (DeCS) e os termos controlados e não controlados do Medical Subject Headings (MeSH): Criança, Menores de Idade, Recém-Nascido, Lactente, (Child, Minors, Infant e Newborn); Transfusão de Sangue; Sangue (Blood Transfusion e Blood); Publicações; Documentos (publications e documents), empregando-se o operador booleano "AND" para realizar as associações de todos os descritores entre si.

A coleta dos artigos foi realizada nos meses de julho e agosto de 2019 e como critério de inclusão para seleção do material utilizou-se: materiais com idiomas em português, Espanhol e Inglês; apenas materiais contidos em periódicos científicos publicados nos anos de 2014 a 2019, sem restrições quanto ao nível de evidência.

Foram encontradas inicialmente 47.293 publicações, no entanto após a aplicação dos critérios de inclusão esse quantitativo reduziu-se para 1.144 artigos. Posteriormente a leitura dos títulos, resumos e objetivos selecionou-se 14 artigos, sendo 2 na BDENF, 10 na LILACS e 2 na MEDLINE. Por não estarem completos ou não responderem à questão da pesquisa 2 artigos foram excluídos, totalizando 12 artigos para a análise e elaboração do Corpus Textual. A Figura 1 representa o fluxograma do processo de seleção dos artigos. 
Figura 1 - Fluxograma do processo de seleção dos artigos. Belém (PA), Brasil, 2019.

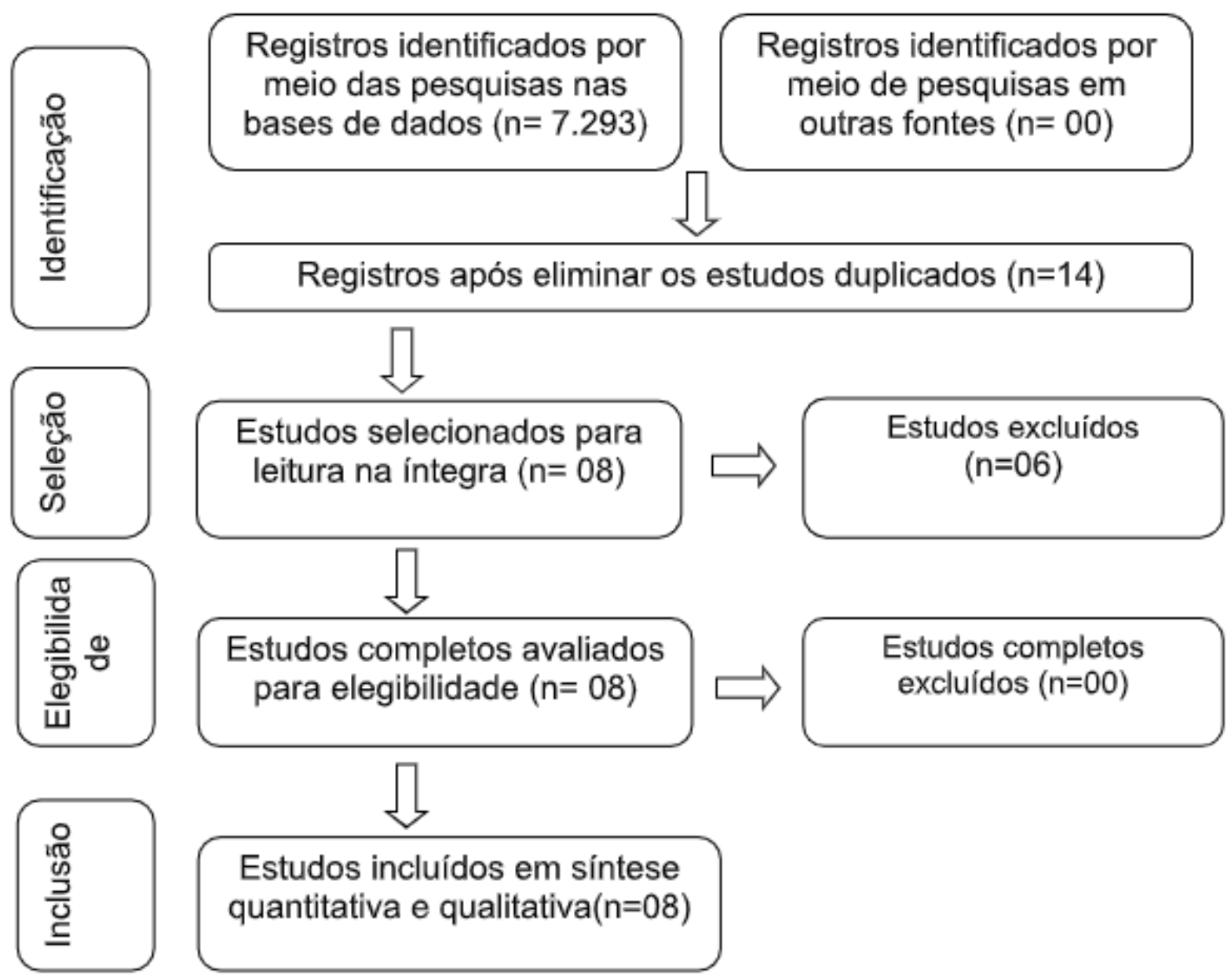

Fonte: Costa PCF, et al., 2019.

Após a seleção do material os dados foram extraídos a partir de um roteiro sistemático com o objetivo de organizar as informações sintetizadas contidas nos artigos selecionados, tais como: código do artigo, título, autor; ano, país, base de dados, objetivo, método e principais conclusões (Quadro 1).

A partir das conclusões dos artigos foi elaborado o corpus textual, para posteriormente utilizar-se o software Interface de $R$ pour lês Analyses Multidimensionnalles de Textes et de Questionnaires (IRAMUTEQ®).

O IRAMUTEQ® foi desenvolvido por Pierre Ratinaud, onde foi utilizado no Brasil a partir 2013. Trata-se de um programa que se ancora no software $\mathrm{R}$ e permite diferentes formas de análises estatísticas sobre corpus textuais e tabelas de indivíduos por palavras (CARMAGO BV e JUSTO AM, 2013).

Para a análise do corpus textual utilizou-se a análise de similitude, que segundo Camargo BV e Justo AM (2013) esta análise é capaz de demonstrar um "no qual é possível representar a ligação entre as palavras do corpus textual, e com isso é permitido inferir como ocorreu a construção textual e os temas de relevância, partindo da coocorrência entre as palavras.

A Figura 2 demonstra um gráfico, onde é apresentada uma interface dos resultados da análise feita no corpus a partir da similitude possibilitando identificar as coocorrências entre as palavras e formações de núcleos de palavras, indicando suas conexões dos termos.

No núcleo central destacam-se as palavras: não, prático, necessário, cuidados neonato e recém-nascido, levando-se a compreender a necessidade de cuidados ao recém-nascido e neonatos internados em uma UTNI. 


\section{RESULTADOS}

Quadro 1 - Caracterização dos artigos incluídos na Revisão Integrativa. Belém (PA), Brasil, 2019.

\begin{tabular}{|c|c|c|c|c|c|c|}
\hline $\begin{array}{c}\text { Código do } \\
\text { Artigo }\end{array}$ & Título & $\begin{array}{l}\text { Autor. Ano. } \\
\text { País }\end{array}$ & $\begin{array}{l}\text { Base de } \\
\text { dados }\end{array}$ & Objetivo & Método & Principais conclusões \\
\hline $\mathrm{A} 1$ & $\begin{array}{l}\text { Práticas de } \\
\text { transfusão em } \\
\text { uma unidade de } \\
\text { terapia intensiva } \\
\text { neonatal em uma } \\
\text { cidade no Brasil }\end{array}$ & $\begin{array}{l}\text { PORTUGAL } \\
\text { CAA, et al. } \\
\text { (2014); }\end{array}$ & LILACS & $\begin{array}{l}\text { Avaliar as práticas } \\
\text { transfusionais em } \\
\text { recém-nascidos em } \\
\text { unidades de terapia } \\
\text { intensiva neonatal de } \\
\text { uma cidade específica. }\end{array}$ & $\begin{array}{l}\text { Estatística } \\
\text { descritiva. }\end{array}$ & $\begin{array}{l}\text { Não houve redução significativa no número de } \\
\text { transfusões por criança no grupo restritivo em } \\
\text { comparação ao grupo liberal. As transfusões } \\
\text { restritivas são um fator de risco independente para } \\
\text { hemorragias peri-intraventriculares e morte. }\end{array}$ \\
\hline $\mathrm{A} 2$ & $\begin{array}{l}\text { Construção e } \\
\text { validação de } \\
\text { checklist para } \\
\text { transfusão } \\
\text { sanguínea em } \\
\text { crianças. }\end{array}$ & $\begin{array}{c}\text { BEZERRA CM, } \\
\text { et al. (2018). }\end{array}$ & LILACS & $\begin{array}{l}\text { Descrever o processo } \\
\text { de construção e } \\
\text { validação de conteúdo } \\
\text { de um checklist para } \\
\text { transfusão sanguínea } \\
\text { em crianças. }\end{array}$ & $\begin{array}{c}\text { Estudo } \\
\text { metodológico. }\end{array}$ & $\begin{array}{l}\text { O checklist para transfusão sanguínea em crianças foi } \\
\text { considerado tecnologia com conteúdo válido para ser } \\
\text { utilizado no ato transfusional desempenhado por } \\
\text { enfermeiros, contribuindo, assim, para segurança } \\
\text { transfusional em crianças. }\end{array}$ \\
\hline A3 & $\begin{array}{l}\text { Anemia } \\
\text { secundaria grave } \\
\text { a excessiva } \\
\text { extração } \\
\text { sanguínea em } \\
\text { recém- } \\
\text { nascidos:uma } \\
\text { chamada para o } \\
\text { cuidado de } \\
\text { neonatologistas. }\end{array}$ & $\begin{array}{l}\text { RIZO DT, et al. } \\
\text { (2018). }\end{array}$ & LILACS & $\begin{array}{l}\text { O objetivo principal } \\
\text { deste estudo é avaliar } \\
\text { as complicações que } \\
\text { surgem devido a } \\
\text { amostras de sangue } \\
\text { repetidas estão } \\
\text { sujeitas a recém- } \\
\text { nascidos durante a } \\
\text { internação. }\end{array}$ & $\begin{array}{l}\text { Uma seção } \\
\text { observacional, } \\
\text { analítico e } \\
\text { descritivo } \\
\text { prospectivo } \\
\text { longitudinal }\end{array}$ & $\begin{array}{l}46,36 \% \text { dos recém-nascidos, mais de } 10 \% \text { do volume } \\
\text { de sangue foi extraído em } 5 \text { dias de idade, o que } \\
\text { aumentou a probabilidade de apresentar anemia } \\
\text { iatrogênica por } 21,71 \text { vezes, transfusões Causando } \\
\text { em } 69,61 \% \text { desses pacientes dos quais } 58,23 \% \\
\text { tinham menos de } 10 \text { dias de vida no momento da } \\
\text { transfusão, além de } 77,3 \% \text { dos recém-nascidos } \\
\text { internados por mais de } 15 \text { dias foram } 24,22 \text { vezes } \\
\text { mais probabilidade de desenvolver este tipo de } \\
\text { anemia. }\end{array}$ \\
\hline A4 & $\begin{array}{l}\text { Reações pós- } \\
\text { transfusionais: } \\
\text { atualizações para } \\
\text { o melhor } \\
\text { desempenho } \\
\text { profissional e } \\
\text { técnico. }\end{array}$ & $\begin{array}{l}\text { GONZALEZ B, } \\
\text { et al. (2017); }\end{array}$ & LILACS & $\begin{array}{l}\text { Avaliar as informações } \\
\text { atualizadas publicadas } \\
\text { na literatura nacional e } \\
\text { internacional sobre } \\
\text { reações pós- } \\
\text { transfusionais. }\end{array}$ & $\begin{array}{c}\text { Estudo } \\
\text { descritivo } \\
\text { transversal. }\end{array}$ & $\begin{array}{l}\text { A revisão da literatura conseguiu compilar as } \\
\text { características mais atualizadas do medicamento } \\
\text { transfusional, para melhor desempenho profissional e } \\
\text { técnico de profissionais pediátricos nas indicações de } \\
\text { transfusões e suas reações adversas. Além de } \\
\text { aumentar a segurança das transfusões em pacientes } \\
\text { pediátricos. }\end{array}$ \\
\hline
\end{tabular}




\begin{tabular}{|c|c|c|c|c|c|c|}
\hline A5 & $\begin{array}{l}\text { Análise da prática } \\
\text { assistêncial em } \\
\text { um hospital } \\
\text { público de ensino: } \\
\text { monitorando } \\
\text { transfussões. }\end{array}$ & $\begin{array}{l}\text { REIS VN, et al. } \\
\qquad(2016)\end{array}$ & MEDLINE & $\begin{array}{l}\text { Analisar o processo de } \\
\text { monitoramento de } \\
\text { gravação transfusão } \\
\text { em um hospital público } \\
\text { de ensino. }\end{array}$ & $\begin{array}{l}\text { Estudo } \\
\text { descritivo e } \\
\text { retrospectivo, } \\
\text { com } \\
\text { abordagem } \\
\text { quantitativa. }\end{array}$ & $\begin{array}{l}\text { Falhas identificadas no processo de monitoramento } \\
\text { de gravação de transfusão, e eles poderiam resultar } \\
\text { em mais eventos adversos relacionados à } \\
\text { administração de componentes sanguíneos. }\end{array}$ \\
\hline A6 & $\begin{array}{l}\text { Perfil } \\
\text { Hematológico dos } \\
\text { neonatos } \\
\text { admitidos em } \\
\text { unidade de } \\
\text { terapia intensiva } \\
\text { neonatal de um } \\
\text { hospital no Sul do } \\
\text { Brasil. }\end{array}$ & $\begin{array}{l}\text { AGUIAR CF, et } \\
\text { al. (2015). }\end{array}$ & LILACS & $\begin{array}{l}\text { Avaliar o hemograma } \\
\text { de admissão destes } \\
\text { pacientes. }\end{array}$ & $\begin{array}{l}\text { Estudo } \\
\text { observacional } \\
\text { com } \\
\text { delineamento } \\
\text { transversal. }\end{array}$ & $\begin{array}{l}\text { O hemograma é um exame rotineiro nas admissões } \\
\text { dos neonatos na Unidade de Terapia Intensiva } \\
\text { Neonatal, que colabora para a alta espoliação } \\
\text { sanguínea e que tende à normalidade nos } \\
\text { parâmetros hematimétricos e do leucograma, com } \\
\text { baixa influência da idade gestacional e peso e possui } \\
\text { uma predisposição à trombocitopenia. }\end{array}$ \\
\hline A7 & $\begin{array}{l}\text { Eventos adversos } \\
\text { e incidentes sem } \\
\text { dano em recém- } \\
\text { nascidos } \\
\text { notificados no } \\
\text { Brasil, nos anos } \\
2007 \text { a } 2013 \text {. }\end{array}$ & $\begin{array}{l}\text { LANZILLOTTI } \\
\text { LS, et al. (2016). }\end{array}$ & MEDLINE & $\begin{array}{l}\text { Analisar os eventos } \\
\text { adversos e outros } \\
\text { incidentes que não } \\
\text { causaram danos em } \\
\text { recém-nascidos até } 28 \\
\text { dias de vida, } \\
\text { notificados no Sistema } \\
\text { de Notificações para a } \\
\text { Vigilância Sanitária } \\
\text { (NOTIVISA) nos anos } \\
\text { de } 2007 \text { a } 2013 \text {. }\end{array}$ & $\begin{array}{l}\text { Estudo } \\
\text { quantitativo, } \\
\text { descritivo, } \\
\text { retrospectivo, } \\
\text { com análise } \\
\text { de dados } \\
\text { secundários. }\end{array}$ & $\begin{array}{l}\text { O estudo desvela notificações de eventos adversos e } \\
\text { de incidentes que não causaram dano, provocando } \\
\text { discussões do que efetivamente é considerado dano } \\
\text { para aquele que faz a notificação. O desafio para o } \\
\text { NOTIVISA é o seu aprimoramento que, como nos } \\
\text { demais sistemas de informação, decorrem de: uso, } \\
\text { crítica e relação com os usuários - notificadores e } \\
\text { interessados, tais como as instituições de pesquisa e } \\
\text { ensino. }\end{array}$ \\
\hline A8 & $\begin{array}{l}\text { Avaliação da dor } \\
\text { durante coleta de } \\
\text { sangue em } \\
\text { crianças sedadas } \\
\text { e submetidas à } \\
\text { ventilação } \\
\text { mecânica. }\end{array}$ & $\begin{array}{l}\text { DANTAS LVRP, } \\
\text { et al. (2016). }\end{array}$ & LILACS & $\begin{array}{l}\text { Avaliar a dor e } \\
\text { observar parâmetros } \\
\text { fisiológicos em } \\
\text { crianças sedadas e } \\
\text { submetidas à } \\
\text { ventilação mecânica } \\
\text { durante um } \\
\text { procedimento de rotina }\end{array}$ & $\begin{array}{c}\text { Estudo } \\
\text { observacional. }\end{array}$ & $\begin{array}{l}\text { Avaliação da dor com uso de escalas padrão, como a } \\
\text { Escala Face, Legs, Activity, Cry and Consolability, e } \\
\text { observação de parâmetros fisiológicos, deve ser } \\
\text { realizada rotineiramente para melhorar o manejo da } \\
\text { dor nas unidades de terapia intensiva pediátricas. }\end{array}$ \\
\hline
\end{tabular}

Fonte: Costa PCF, et al., 2019. 
Já interligado a este núcleo encontra-se a formação de um grupo de palavras, as quais se destacam: transfusão, relação, estudo e erro, podendo inferir a partir deste agrupamento de palavras que há a possibilidade de erros ao longo do ato transfusional em UTNI.

Em um agrupamento mais distante podemos encontrar as seguintes palavras em destaque: informação, sistema, pesquisa e especialmente. Levando a conclusão a partir deste núcleo de palavras sobre a necessidade de um sistema de informação e novas pesquisas para nortear os cuidados transfusionais que ocorrem nas UTIN's.

Outros grupos de palavras em destaque são: notificação, discussão, incidente, adverso e dano. Este agrupamento de palavras leva a considerar que nos artigos bases desta revisão integrativa os autores destacam que há a necessidade de se discutir e fortalecer a discussão do sistema de notificação vigente, pois os danos e efeitos adversos podem ser motivos de complicações permanentes aqueles que se submetem a terapêutica do sangue, tais como os pacientes internados em Unidades de terapia intensiva neonatal.

Figura 2 - Gráfico de análise de similitude, software IRAMUTEQ®. Belém (PA), Brasil, 2019.

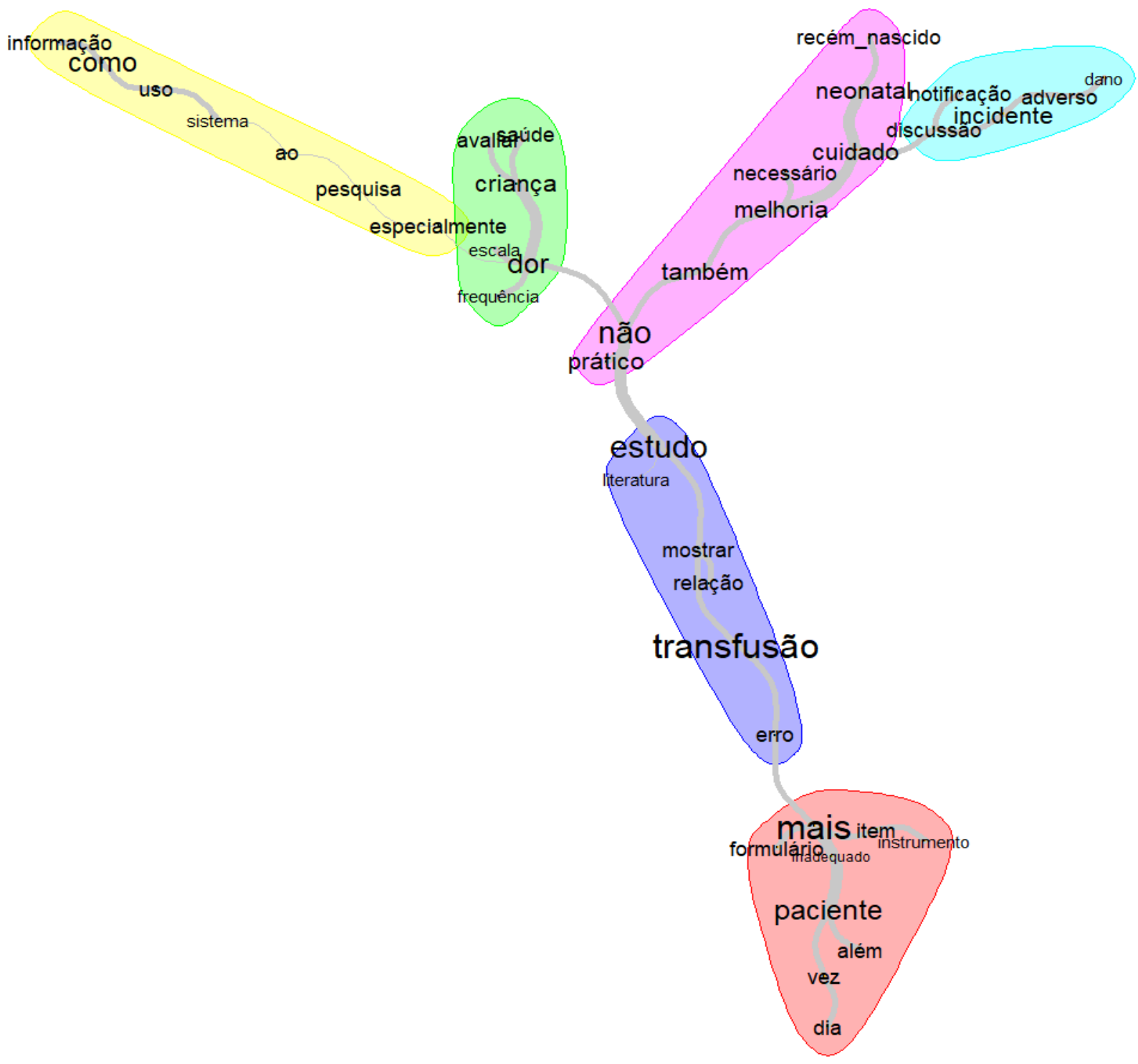

Fonte: Costa PCF, et al., 2019. 


\section{DISCUSSÃO}

Nas hemotransfusões realizadas em Unidades de Tratamento Intensivo Neonatal, o principal componente utilizado é o concentrado de hemácias, observando-se que as principais notificações de reações durante a utilização do sangue, está relacionado ao uso de hemácias na terapêutica, tendo como principais reações a febril não hemolítica e a reação alérgica, em sua maioria eventos considerados leves e uma minoria classificado como moderado a grave (GRANDI JL, et al., 2017).

$\mathrm{O}$ ato transfusional é importante dentro da UTIN, já que trata de um procedimento que busca beneficiar os clientes, assim como fomentar os bancos de sangue, além de ser um processo que pode ser decisivo no quadro de recuperação ou mudança de quadro do paciente. Os componentes do sangue e seus derivados transfundidos são suporte para a terapia de diversas doenças assim como apoio à transplantes, cirurgias e quimioterapias, sendo dessa forma essenciais e insubstituíveis (CHEREM EO, 2018; SILVA AS, 2011; SOUZA FR e CERQUEIRA ETV, 2018).

Em unidades hospitalares, principalmente, em clínicas cirúrgicas e Unidades de Terapia Intensiva, seja ela, neonatal, infantil ou adulta é rotineiro o uso de hemocomponentes, tais como o concentrado de hemácias, sendo considerada uma medida de suporte de vida essencial aos cuidados dispensados aos indivíduos (FIDLARCZYK D e FERREIRA SS, 2008).

No entanto, é importante ressaltar que o ato transfusional envolve um procedimento sujeito a riscos, assim como outras intervenções terapêuticas, podendo ocasionar complicações agudas ou tardias, como a possível transmissão de agentes infecciosos e outras complicações de cunho clínico, como as reações transfusionais, que podem ser definidas como uma resposta indesejável no indivíduo, em associação à administração do sangue ou hemocomponente. Pode ocorrer devido a um incidente do ciclo do sangue ou interação entre receptor e o hemoderivado, sendo este um produto biologicamente ativo (SILVA EM, et al., 2017; MANUAL DE HEMOVIGILÂNCIA, 2013).

A segurança transfusional e a qualidade dos produtos hemoterápicos são preocupações contínuas dos serviços especializados em hemoterapia e hematologia, assim como, das autoridades sanitárias e da sociedade, tornando a segurança nos procedimentos que envolvem o sangue, elemento primordial nos sistemas de saúde (SCHÖNINGER N e DURO CLM, 2010).

Levando em consideração os materiais bibliográficos analisados e seus resultados, também encontramos indícios de falhas dentro de tal processo, uma vez que, há um deficit no quesito conhecimento científico da prática do ato transfusional, fato evidenciado na fala de Silva EM, et al. (2017), no qual incita que o a transfusão, apesar de estar presente na maioria das clínicas, com grande ou pequena proporção, os profissionais que executam a atividade com grande frequência, possui certa insegurança quanto à informação (SILVA EM, et al., 2017). E ainda por Ângulo IL (2007) ao evidenciar a complexidade do processo transfusional e a necessidade de profissionais habilitados e capacitados tecnicamente quanto cientificamente na garantia da segurança da hemotransfusão (ÂNGULO IL, 2007). Tal carência pode estar relacionada ao fato de haver poucos dados e evidências científicas acerca dessa temática, como destacado na fala de Portugal CAA, et al. (2014) ao afirmar que mesmo sendo uma prática de grande abrangência, encontram-se poucos materiais científicos quanto a condição de transfusão de hemácias (PORTUGAL CAA, et al., 2014).

Tendo isso em vista, é necessário que haja discussão e indagações, a fim de fortalecer os parâmetros de investigação, gerando descobertas científicas, sobre os motivos que ocasionam os efeitos adversos durante ou após o ato da transfusão e fomentando cada vez mais a capacitação dos profissionais, podendo criar novos métodos para a prevenção aos erros envolvendo a equipe de transfusão, pois como fala de Bezerra CM, et al. (2018) de que, a maior parte dos erros transfusionais está nos quesitos: Identificação do paciente, procedimento de coleta de amostra de sangue ou devido a checagem inadequada antes da instalação do hemocomponente na beira do leito (BEZERRA CM, et al., 2018).

Desta forma, a educação deve ser continua nos serviços de saúde, tornando isso uma ferramenta essencial na qualificação da assistência segura prestada pelos profissionais da saúde. A maioria dos 
profissionais ainda possuem um conhecimento superficial sobre reações adversas na assistência hemoterápica, mas conseguem entender que o reconhecimento de eventos adversos associados a transfusão faz parte de sua assistência ao seu cliente, principalmente se falhas nesta assistência não forem observadas no tempo necessário. Evidencia-se também que há muita subnotificação de eventos adversos relacionados à prática em hemoterapia, sendo isto um fator importante de atuação da educação continuada de serviços de saúde (MOREIRA IA, et al., 2015).

Ademais, cientes de tal atraso é perceptível a necessidade da criação de novas tecnologias e instrumentos os quais levem, tanto à otimização e aprimoramento, quanto à eficácia da terapia transfusional em neonatais, como o check-list criado por especialistas brasileiros, no qual mede o nível de conhecimento de acordo com as categorias e especialidades de profissionais que estão incluídos dentro do âmbito da terapia transfusional em UTIN's. Uma vez que, danos acometidos durante a terapia transfusional e situações de risco podem ser evitados por meio do conhecimento científico e habilidade profissional, garantindo um processo eficiente (BEZERRA CM, et al., 2018; SILVA EM, et al., 2017). E ressaltado por Souza WFR e Cerqueira ETV (2018) tornando imprescindível detectar, investigar e notificar de forma sistemática as hemotransfusões na redução de riscos e danos aos pacientes, através de medidas corretivas e preventivas no processo do ciclo do sangue (SOUZA WFR e CERQUEIRA ETV, 2018).

\section{CONSIDERAÇÕES FINAIS}

A revisão integrativa da literatura permitiu agregar as publicações e identificar que a temática transfusão de sangue em unidades de terapia intensiva neonatais é discutida nacionalmente e mundialmente nas publicações, e que há um reconhecimento da importância da discussão desta terapêutica nos cuidados neonatais, porém, evidenciou-se que há uma preocupação quando abordados sobre os riscos em se realizar as transfusões em ambientes como as unidades de cuidados intensivos neonatais. Sendo claramente recomendados que há uma necessidade de monitoramento e acompanhamento de incidentes relacionados ao uso de sangue nestas unidades. Concluindo-se que há relevância em continuar as pesquisas relacionadas a esta temática e que outros estudos sejam feitos para o fortalecimento deste conhecimento.

\section{REFERÊNCIAS}

1. ÂNGULO IL. Hemoterapia moderna, práticas antigas. Rev Bras Hematol Hemoter. 2007,29(2):108.

2. BEZERRA CM, et al. Construção e validação de checklist para transfusão sanguínea em crianças. Revista Brasileira de Enfermagem. 2018; 71(6): 3196-202.

3. BOWMAN J. The management of hemolytic disease in the fetus and newborn. Semin Perinatol 1997; 21:39-44.

4. BRASIL. Ministério da Saúde. Hemovigilância. Manual Técnico de Hemovigilância -Investigação das Reações Transfusionais Imediatas e Tardias Não Infecciosas. Brasília (DF): Anvisa; 2013.

5. BRASIL, Ministério da Saúde. Agência Nacional de Vigilância Sanitária. RDC n. 34, de 11 de junho de 2014. Dispõe sobre as boas práticas no ciclo do sangue. Brasília: MS; 2014.

6. CAMARGO BV, JUSTO, AM. Tutorial para uso do software de análise textual IRAMUTEQ: Universidade Federal de Santa Catarina, Florianopolis, 2013. 18 p.

7. CHEREM EO. O conhecimento do enfermeiro sobre a hemotransfusão em uma unidade de terapia intensiva neonatal. Dissertação (Pós-graduação em atenção a Saúde Maternoinfantil) - Faculdade de Medicina. Universidade Federal Fluminense, Rio de Janeiro, 2015.

8. CHEREM EO, et al. Processo de terapia transfusional em unidade de terapia Intensiva neonatal: o conhecimento do enfermeiro. Texto Contexto Enfermagem, 2018; 27(1):e1150016.

9. CHEREM EO, et al. Cuidado pós-transfusional na unidade de terapia intensiva neonatal. Rev baiana enferm. 2016; 30(4):1-8.

10. DINIZ EMA, et al. Uso de sangue, hemocomponentes e hemoderivados no recém-nascido. J Pediatr, 2001;77(supl.1):104-14.

11. DUARTE ED, et al. Práticas cuidadoras que favorecem a integralidade do cuidado ao recém-nascido de alto risco: revisão sistemática. Rev Eletr Enferm. 2010; 12(3). 
12. FERREIRA O, et al. Avaliação do conhecimento sobre hemoterapia e segurança transfusional de profissionais de Enfermagem. Rev Bras Hematol Hemoter. 2007; 29(2): 160-7.

13. FIDLARCZYK D, FERREIRA SS. Enfermagem em Hemoterapia. Rio de Janeiro: MedBook; 2008.

14. FREITAS BAC, FRANCESCHINI SCC. Fatores associados à transfusão de concentrado de hemácias em prematuros de uma unidade de terapia intensiva. Rev Bras Ter Intensiva. 2012; 24(3): 224-9.

15. GRANDI JL, et al. Frequência dos incidentes transfusionais imediatos em receptores de hemocomponentes. Vigil sanit Debate. 2017; 5(2):83-8.

16. KUTNER JM, et al. Manual de orientação para o uso de sangue, hemocomponentes e aféreses terapêuticas. 3rd ed. São Paulo: Atheneu; 2003.

17. MENDES NM, SOUZA SROS. Dimensões da transfusão de hemocomponentes em unidade de terapia intensiva de adulto. Rev Hospital Universitário Pedro Ernesto. 2011; 10(supl. 1):83-92.

18. MOREIRA IA, et al. Conhecimento dos profissionais de saúde sobre eventos adversos em unidade de terapia intensiva. Rev enferm UERJ. 2015; 23(4):461-7.

19. MURRAY NA, ROBERTS IA. Neonatal transfusion practice. Arch Dis Child Fetal Neonatal. Ed. 2004; 89(2):101-7.

20. PORTUGAL CAA, et al. Transfusion practices in a neonatal intensive care unit in a city in Brazil. Revista brasileira hematol hemoter. 2014; 36(4): 245-49.

21. PHILIP AG. The rise and fall of exchange transfusion. Neo Reviews. 2003; 4: 169-74.

22. SCHÖNINGER N, DURO CLM. Atuação do enfermeiro em serviço de hemoterapia. Ciênc Cuid Saúde. 2010; 9(2):317-24.

23. SHANNON KM, et al. Recombinant human erythropoietin stimulates erythropoiesis and reduces erythrocyte transfusions in very low birth weight preterm infants. Pediatrics. 1995; 95 (1): 1-8.

24. SILVA AS, et al. Percepções e mudanças na qualidade de vida de pacientes submetidos à hemodiálise. Revista Brasileira de Enfermagem, 2011; 64(5): 839-44.

25. SILVA EM, et al. Desafios da enfermagem diante das reações transfusionais. Revista de enfermagem UERJ, Rio de Janeiro, 2017; 25:e11552.

26. SILVA KFN, et al. A prática transfusional e a formação dos profissionais de saúde. Rev. Bras. Hematol. Hemoter. 2009; 31(6): 421-426.

27. SOUZA WFR, CERQUEIRA ETV. A atuação do enfermeiro na gestão do cuidado em reações transfusionais. Rev Eletro Acervo em Saúde. 2018.

28. SOUZA GF, et al. Good nursing practices in the intensive care unit: care practices during and after blood transfusion. Reme - rev min enferm. 2014; 18(4): 947-54.

29. TOREZAN G, SOUZA EN. Transfusão de hemoderivados: os enfermeiros estão preparados para o cuidado peritransfusional? Rev Enferm UFPE on line. 2010; 4(2): 658-65. 